\title{
Individual fates of mesenchymal stem cells in vitro
}

\author{
Axel Krinner', Martin Hoffmann', Markus Loeffler², Dirk Drasdo 1,3 and Joerg Galle*1
}

\begin{abstract}
Background: In vitro cultivated stem cell populations are in general heterogeneous with respect to their expression of differentiation markers. In hematopoietic progenitor populations, this heterogeneity has been shown to regenerate within days from isolated subpopulations defined by high or low marker expression. This kind of plasticity has been suggested to be a fundamental feature of mesenchymal stem cells (MSCs) as well. Here, we study MSC plasticity on the level of individual cells applying a multi-scale computer model that is based on the concept of noise-driven stem cell differentiation.
\end{abstract}

Results: By simulation studies, we provide detailed insight into the kinetics of MSC organisation. Monitoring the fates of individual cells in high and low oxygen culture, we calculated the average transition times of individual cells into stem cell and differentiated states. We predict that at low oxygen the heterogeneity of a MSC population with respect to differentiation regenerates from any selected subpopulation in about two days. At high oxygen, regeneration becomes substantially slowed down. Simulation results on the composition of the functional stem cell pool of MSC populations suggest that most of the cells that constitute this pool originate from more differentiated cells.

Conclusions: Individual cell-based models are well-suited to provide quantitative predictions on essential features of the spatio-temporal organisation of MSC in vitro. Our predictions on MSC plasticity and its dependence on the environment motivate a number of in vitro experiments for validation. They may contribute to a better understanding of MSC organisation in vitro, including features of clonal expansion, environmental adaptation and stem cell ageing.

\section{Background}

The generation and maintenance of replenishing tissues relies on an appropriately regulated balance between selfrenewal and differentiation within a relatively small population of adult stem cells. According to the common stem cell paradigm this balance can be explained assuming a strict differentiation hierarchy and irreversible fate decisions [1,2]. However, the organisation of stem cell populations is strongly influenced by environmental factors such as specific cell-cell interactions, growth factor and oxygen supply, as well as the geometry and mechanical properties of the local environment [3,4]. Accordingly, it has been suggested that stemness represents a particular regulatory cell state rather than an entity and that this state may be approached in principle by any cell $[5,6]$. Supporting these ideas, recent experimental results in hematopoietic systems demonstrated that stem cell populations can actually regenerate from more differentiated

* Correspondence: galle@izbi.uni-leipzig.de

1 Interdisciplinary Centre for Bioinformatics, University of Leipzig, Haertelstr. 1618, 04107 Leipzig, Germany

Full list of author information is available at the end of the article subpopulations $[7,8]$. Currently, there is an ongoing debate on fundamental dynamics underlying this kind of cell plasticity. In particular, it remains open whether dedifferentiation is prerequisite to lineage changes. A thorough understanding of this phenomenon is expected to make an important contribution to the development of novel therapeutic strategies for treating degenerative disease, injury and neoplasia.

Mesenchymal stem cells (MSCs) are multi-potent cells that persist in adult life in some tissue types, such as bone-marrow stroma, fat, skeletal muscle, and synovium without loosing their capacity to proliferate and differentiate $[9,10]$. Under appropriate culture conditions, they can multiply and transform into specialized cell types in vitro. Plasticity of MSCs of the 3T3 T type linked to dedifferentiation has already been demonstrated in the Eighties [11]. More recently, also differentiation of adult human MSC was found to be at least partially reversible [12]. In fact plasticity has been suggested to represent a fundamental feature of MSC [13].

Recently, we have introduced a multi-scale computer model of MSC expansion, lineage commitment and dif- 
ferentiation which consistently explains a panel of experimental results regarding the oxygen dependence of these processes and predicts optimal culture conditions [14]. This model utilises the concept of noise-driven stem cell differentiation [15] which is based on the functional stem cell approach to tissue organisation by Roeder \& Loeffler $[5,16]$. According to this concept, MSC plasticity bases on permanent fluctuations of the differentiation state of each individual cell, which enables more differentiated cells to re-gain stem cell properties and subsequently to switch lineage (details see below).

Here we aim at quantitative predictions on MSC organisation in vitro based on our former results. For this purpose we performed "experiments in silico" using our novel multi-scale model. We monitored the fates of individual MSCs under different culture conditions. Linking intracellular regulation of the differentiation state to cell biomechanics our computer simulations provide insight into possible mechanisms of how cell-cell and cell-substrate interaction can affect stem cell functionality. Thereby, our computer simulations were designed as MSC protocols in silico such that they can be directly tested in vitro.

In the following we first give a brief description of the model of MSC organisation in vitro introduced by Krinner et al. [14] and provide the experimentally validated data set used throughout this study. Subsequently, we present our simulation results on MSC plasticity and discuss the potential and the limits of our approach.

\section{Model}

\section{Noise-driven differentiation dynamics}

In our model cell differentiation is defined as the loss of stem cell properties. Cell differentiation is quantified by a continuous state variable $\alpha$ that can adopt values between zero (full stem cell competency) and one (completely differentiated cell). Each value of $\alpha$ may represent a set of regulatory network activation patterns. From the molecular point of view, $\alpha$ may depend on the abundance and sub-cellular localization of proteins and RNAs, as well as other types of signalling and metabolic molecules [17]. Cell differentiation is assumed to occur independently of cell proliferation [18].

The model assumes that each cell's $\alpha$-value fluctuates randomly with a state dependent noise amplitude $\sigma(\alpha)$. From its current $\alpha$ value a cell adopts a new value $\alpha^{\prime}$ with a transition rate R. $\alpha^{\prime}$ is drawn from a Gaussian distribution $\mathrm{p}\left(\alpha^{\prime} \mid \alpha\right)$, centred around $\alpha$ with standard deviation $\sigma(\alpha)$. According to this assumption, cells tend to accumulate in low noise states. The state dependence of $\sigma(\alpha)$ is further assumed to be determined by the environment. Hence, a differentiation-inducing environment reduces noise in high $\alpha$ states causing an accumulation of cells in differentiated states (see Figure 1a).
MSC differentiation involves lineage-priming [19]. This process implies particular cellular decisions, which can be modelled considering a second state variable [14]. We here assume that differentiation and de-differentiation dynamics do not depend on these decisions. However, a switch from one into another specific lineage may require a defined degree of stemness as suggested for the chondrogenic lineage [14]. In this case, differentiation stabilises lineages and the described capability of dedifferentiation is synonymous with MSC plasticity in general. We here focus on that kind of MSC plasticity.

An important environmental factor during MSC expansion is oxygen $[14,20]$. In our model, we assume an oxygen dependent control of the state fluctuations. Increasing oxygen tension reduces the state fluctuations in differentiated states, thereby inducing unspecific differentiated, non-proliferative cells. This was implemented assuming the following dependence of the noiseamplitude $\sigma(\alpha)$ on the oxygen tension $\mathrm{pO}_{2}$ :

$\sigma(\alpha)=\sigma_{0}\left(1-\alpha f\left(p O_{2} / p O_{2}^{\max }\right)\right)$ with $f(x)=\frac{x^{n}}{x^{n}+k^{n}}$

where $\sigma_{0}$ denotes the fluctuation strength in stem cell states and $f$ is a Hill function approaching 0 and 1 at low and high $\mathrm{pO}_{2}$, respectively.

Cell proliferation is assumed to depend on the differentiation state $\alpha$ of a cell. In our model, it is restricted to intermediate differentiation states $\alpha_{\mathrm{p}}$ with: $0<\alpha_{\mathrm{s}}<\alpha_{\mathrm{p}}<\alpha_{\mathrm{d}}$ $<1$ (Figure 1b). These states are termed 'progenitor states' in the following. For these proliferative states we assume an identical doubling rate $r=1 / \tau$ and average growth time $\tau$. 'Stem cells' $\left(\alpha<\alpha_{\mathrm{s}}\right)$ and 'differentiated cells' $\left(\alpha>\alpha_{\mathrm{d}}\right)$ do not proliferate. The state fluctuations cause the cells to switch frequently between proliferative and non-proliferative state, which results in an effective average growth time larger than $\tau$.

\section{Individual cell-based model (IBM)}

In order to simulate the spatio-temporal dynamics of MSC populations we use an IBM where the cells are modelled as elastic adhesive spheres [21]. We assume that the cell volume in suspension cannot be smaller than a minimum value $V_{0}$. A cell can move actively by migration and passively by being pushed, it can deform, adhere to other cells or a substrate, and it can grow and divide. A proliferating cell divides if its volume has grown to twice the volume $\mathrm{V}_{0}$.

Assuming that cells can approximately be described by an isotropic homogenous elastic solid, cell-cell and cellsubstrate interaction are modelled by a modified Hertz- 

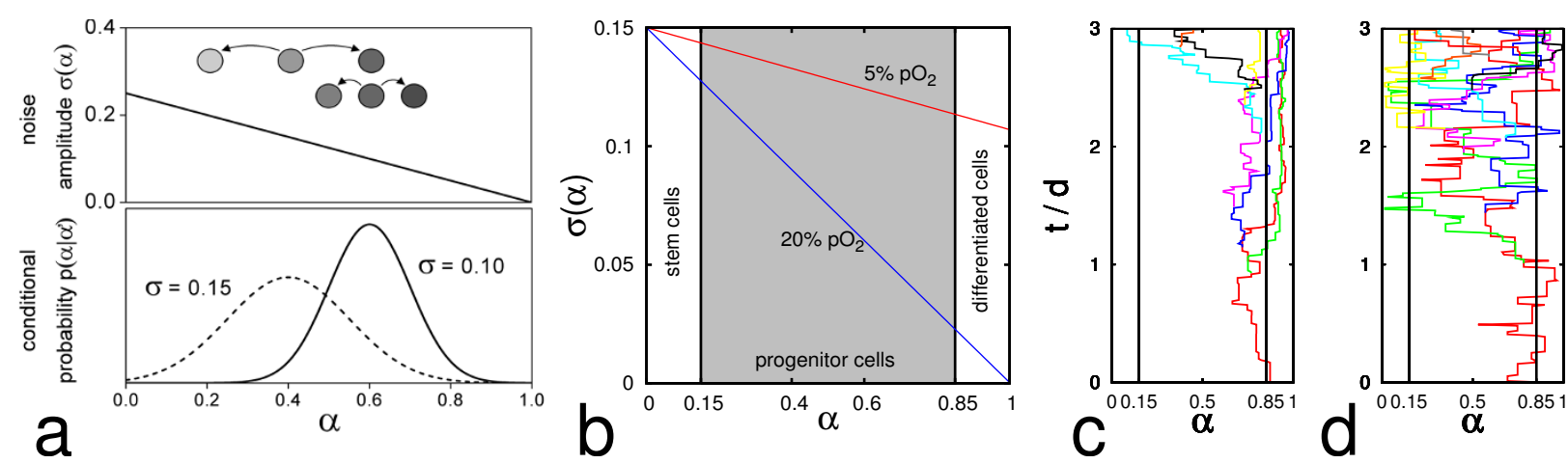

Figure 1 Noise driven differentiation dynamics. a) Modelling fluctuations of the differentiation state a. Upper panel: A decrease of the noise amplitude $\sigma(a)$, i.e. the width of the Gaussian conditional probability function $p\left(a \mid a^{\prime}\right)$, with a results in an average drift to higher values of a. Lower panel: $p\left(a \mid a^{\prime}\right)$ for $a^{\prime}=0.4$ and $a^{\prime}=0.6$. b) Noise amplitude for in vitro MSC expansion at $5 \%$ and $20 \% \mathrm{pO}_{2}$. Proliferative states $a_{p}$ are shown in grey. c), d) Genealogies of expanding clones in the $a$-space of differentiation at $20 \%$ and at $5 \% \mathrm{pO}_{2}$, respectively. a-trajectories of progeny arising from cell division are shown in individual colours.

Potential, consisting of the classic Hertz-Potential and an adhesion term [22]:

$V_{\text {ges }}=V_{\text {Hertz }}+V_{\text {adh }}=\frac{8}{15}\left(\frac{1-v_{1}^{2}}{E_{1}}+\frac{1-v_{2}^{2}}{E_{2}}\right)^{-1} \sqrt{\frac{R_{1} R_{2}}{R_{1}+R_{2}}} \delta^{5 / 2}-\varepsilon_{12} \pi \frac{R_{1} R_{2}}{R_{1}+R_{2}} \delta$.

In the first term on the right hand side $v_{\mathrm{i}}$ denotes the Poisson's ratio of the interaction partner $i(i=1,2), E_{i} i t s$ Young modulus, $R_{i}$ its radius (substrate radius $R=\infty$ ) and $\delta$ the surface deformation. The second term models adhesion proportional to the Hertz contact area, where $\varepsilon_{12}$ is the anchorage given as adhesion energy per unit area.

Cell proliferation is modelled assuming a two phase cell cycle: During the interphase, a cell doubles its volume by stochastic increments. During the mitotic phase, a cell divides into two daughter cells of equal volume. This growth process results in an approximately $\Gamma$-distributed growth time $\tau$ of the cells [23]. A cell undergoes a growth arrest if the sum of the deformation forces on it exceeds a critical value $\mathrm{F}_{\mathrm{c}}$.

We simulate cell motion by using a Langevin equation for each cell [21]. The small Reynolds numbers in the regime of single cells allows us to neglect inertia, leading to a linear system of stochastic equations for the cell displacements. Thereby, the displacement $d x_{i}$ of cell $i$ is given by:

$$
\gamma_{i s} \frac{d \mathbf{x}_{i}}{d t}+\sum_{j} \gamma_{i j}\left(\frac{d \mathbf{x}_{i}}{d t}-\frac{d \mathbf{x}_{j}}{d t}\right)=\mathbf{F}_{i}^{s t o c h}+\sum_{j} \mathbf{F}_{i j}^{H e r t z}
$$

where the sums run over all neighbouring cells $j$ in direct contact to cell i. $\mathbf{F}_{\mathrm{ij}}$ Hertz denotes the Hertz force between cell $i$ and cell $j$ and $F_{i}$ stoch the stochastic Langevin force on cell $i$. The friction coefficients $\gamma_{\text {is }}$ and $\gamma_{i j}$ describe friction between cell $i$ and the substrate and between cell $i$ and cell $j$, respectively. These coefficients are assumed to be proportional to the respective contact areas. Details can be found in [14].

\section{Master equation approach}

In addition to the IBM we pursue a theoretical population dynamics model as previously described [15]. Here, we use this model for studying the population average of dynamic properties of individual cells; therefore proliferation is not included. The model is then equivalent to a master equation for a Markov process [24] describing the dynamics of the average number of cells $\mathrm{N}(\alpha)$ in state $\alpha$ :

$$
\frac{1}{R} \frac{\partial N(\alpha(t))}{\partial t}=\int_{0}^{1} p(\alpha \mid \bar{\alpha}) N(\bar{\alpha}, t) d \bar{\alpha}-N(\alpha, t)
$$

with transition probability $p(\alpha \mid \bar{\alpha}) \propto \exp \left(-(\alpha-\bar{\alpha})^{2} / 2 \sigma^{2}(\bar{\alpha})\right)$ and constant randomization rate R. Transition times $\vartheta(\alpha)$ from an initial $\alpha$ into the regimes of stem cells $\left(\alpha<\alpha_{\mathrm{s}}\right)$ or differentiated cells $\left(\alpha>\alpha_{d}\right)$ were computed using an absorbing boundary approach [24].

\section{Model parameters}

Our model of MSC differentiation dynamics depends on parameters describing intracellular regulation; the randomization rate $\mathrm{R}$, the stem cell state fluctuation strength $\sigma_{0}$, the parameters of the Hill function ( $\mathrm{n}$ and $\mathrm{k}$ ) and those specifying the proliferation rate $\left(r\right.$ and $\alpha_{s}$ with $\left.\alpha_{d}=1-\alpha_{s}\right)$. 
The IBM of spatio-temporal organisation of growing MSC populations depends on parameters specifying cellcell and cell-substrate interaction, as the Poisson's ratio, the Young modulus, and the friction constants. Combining these models in a particular application one has to adjust a large parameter set.

Recently, we have applied the combined multi-scale model to ovine MSC expansion at low (5\%) and high (20\%) oxygen tension [14]. These former investigations enable us to use an experimentally validated set of model parameters in the present study. These parameters are summarized in Table 1. We used this parameter set in all simulations if not further specified.

\section{Results}

\section{Monitoring individual cell fates}

Using the IBM the fates of individual cells in growing populations can be monitored. We simulated individual $\alpha$-trajectories and compared the cell differentiation dynamics at low $(5 \%)$ and high $(20 \%)$ oxygen concentrations. The genealogies of two selected clones in $\alpha$ space

Table 1: Parameter set used in the simulations

\begin{tabular}{|c|c|}
\hline Parameter & Value \\
\hline \multicolumn{2}{|l|}{ Intracellular regulation } \\
\hline Randomization Rate $\mathrm{R}$ & $2.5 \times 10^{4} \mathrm{~s}^{-1}$ \\
\hline Stem Cell State Fluctuation Strength $\sigma_{0}$ & 0.15 \\
\hline Hill Coefficient $\mathrm{n}$ & 5 \\
\hline Dissociation Constant k & 0.3 \\
\hline Differentiation Threshold $a_{s}\left(a_{d}=1-a_{s}\right)$ & 0.85 \\
\hline \multicolumn{2}{|l|}{ Spatio-temporal organisation } \\
\hline Minimal Cell Radius $\mathrm{R}_{0}$ & $5 \mu \mathrm{m}$ \\
\hline Minimal Cell Volume $\mathrm{V}_{0}$ & $\mathrm{~V}\left(\mathrm{R}_{0}\right)$ \\
\hline Proliferation Rate $r=1 / \tau$ & 1.9/day \\
\hline Young Modulus E & $450 \mathrm{~Pa}$ \\
\hline Contact Inhibition Threshold $\mathrm{F}_{\max }$ & $1 \times 10^{-9} \mathrm{~N}$ \\
\hline Poisson's Ratio v & 0.4 \\
\hline Friction Coefficients $\gamma_{\mathrm{ij}}, \gamma_{\mathrm{is}}$ & $\begin{array}{l}3 \times 10^{7} \mathrm{Ns} / \mathrm{m}^{3} \\
1 \times 10^{11} \mathrm{Ns} / \mathrm{m}^{3 *}\end{array}$ \\
\hline Cellular Diffusion Coefficient $\mathrm{D}_{\text {Cell }}$ & $4 \times 10^{-12} \mathrm{~cm}^{2} / \mathrm{s}$ \\
\hline Cell-Cell Anchorage $\varepsilon$ & $6 \times 10^{-5} \mathrm{~N} / \mathrm{m}$ \\
\hline Cell-Plane Anchorage $\varepsilon$ & $6 \times 10^{-5} \mathrm{~N} / \mathrm{m}$ \\
\hline Quiescence Threshold $F_{q}$ & $10 \mathrm{~N} / \mathrm{m}^{2}$ \\
\hline
\end{tabular}

The parameter set was adjusted using experimental data on the clone size distribution of ovine MSC growing in vitro [14]. * The high substrate friction coefficient $\gamma_{\text {is }}$ was used in 'population regeneration' simulations (Sec. 3.2) in order to study the influence of biophysical properties on stem cell plasticity. are shown in Figure 1c and 1d, for low and high oxygen, respectively.

In order to quantify the degree of plasticity that is inherent in MSCs we calculated the average time required to adopt specific cellular phenotypes. The average transition times of a cell to reach stem cell states $(0.0$ $\left.<\alpha<\alpha_{\mathrm{s}}=0.15\right)$ and differentiated states $\left(\alpha_{\mathrm{d}}=0.85<\alpha<\right.$ 1.0) were calculated as follows: 100.000 cells with $\alpha$-values equally distributed in the interval $[0,1]$ were subjected to state fluctuations. Throughout the simulations cells that reached the specified subpopulation for the first time were counted and histograms about their initial state were derived. From these histograms we calculated the i) average transition times (Figure 2a, b) and ii) the fractions of cells that successfully transferred within a defined time.

Our results demonstrate that at low oxygen a frequent exchange between the subpopulations occurs on a time scale of about 2 days. At high oxygen the average transition time for stem cells into the pool of differentiated cells increases to about 4 days. Transition times for differentiated cells into the stem cell pool at high oxygen are much larger ( $>100$ days), indicating quasi-deterministic cell differentiation behaviour. We confirmed our results using the master equation approach. In Figure $2 \mathrm{c}$ the fraction of cells having entered the stem cell pool at $20 \% \mathrm{pO}_{2}$ is shown as a function of the initial $\alpha$ value and the simulation time. Only in this particular case, the fraction of absorbed cells grows too slowly to calculate the average transition times. In the three other cases, they were computed with high precision (less than $10^{-12}$ of all cells remain to be absorbed).

Since stem cell states are more easily accessible at low oxygen compared to high oxygen we predict MSC plasticity to be more pronounced under these conditions.

In vitro validation of the above results would require single cell tracking of MSCs and techniques to identify the differentiation state of the tracked cells. Currently, considerable effort is taken in order to establish tracking techniques for stem cell systems $[25,26]$. Unfortunately, MSCs are particularly hard to track, because they tend to aggregate; a phenomenon known as mesenchymal condensation $[27,28]$. Thus, in the following we present results on MSC plasticity as seen on the population level which can be validated in simpler experimental setups.

\section{Modelling regeneration of the population structure}

Chang et al. [7] studied how fast the distribution of differentiation marker expression within a cell population regenerates from subpopulations with defined expression level. They performed the following experiment: a population of precursor cells was generated under standard conditions and characterised by the expression level of a particular differentiation marker. Subpopulations of cells 


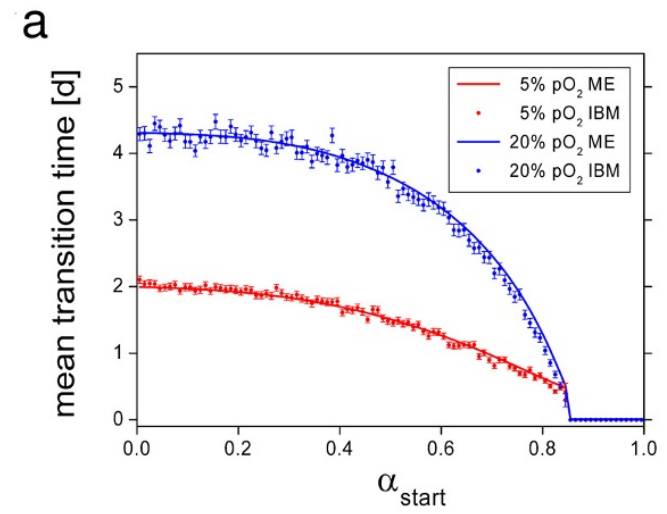

b

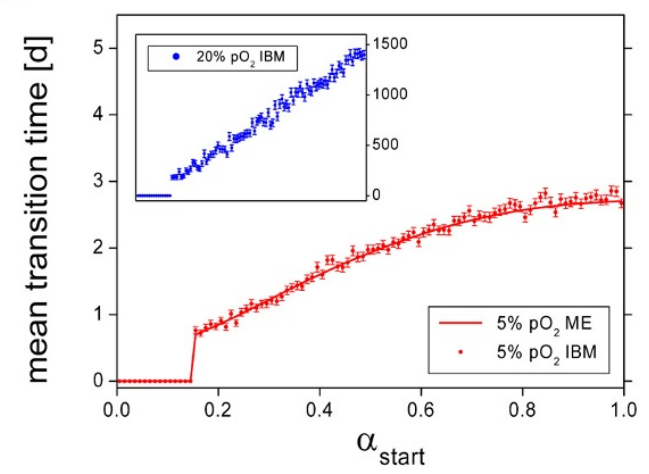

C

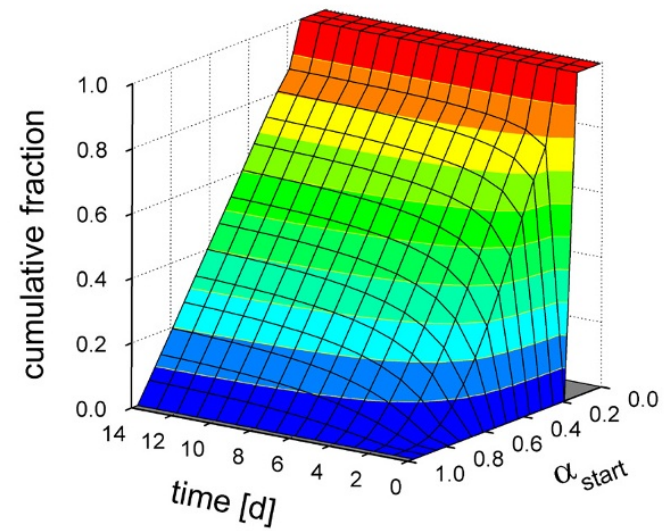

Figure 2 Simulated individual cell dynamics. a), b) Mean transition times calculated using IBM and the master equation approach (ME) to reach (a) differentiated states and (b) stem cell states at $5 \%$ (red) and at 20\% $\mathrm{pO}_{2}$ (blue). The transition time to stem cell states at $20 \% \mathrm{pO}_{2}$ was not calculated using the ME because the fraction of cells that have reached stem cell states once converged too slowly (see c). Symbols: IBM results, Lines: Master equation. (c) Fraction of cells that reach stem cell states at $20 \% \mathrm{pO}_{2}(\mathrm{ME})$ as a function of initial a and simulation time.

with defined expression levels of the differentiation marker were separated. These subpopulations were cultivated under standard conditions and regeneration of the distribution of expression levels in the population was monitored over time by FACS.

We simulated this population regeneration experiment as follows: Starting from a population that was grown at low density, i.e. which shows no signs of contact inhibition of growth, we selected 200 stem cells and 200 differentiated cells and followed their development over 5 days in secondary cultures. In order to characterise the environmental dependence of the regeneration process, we compared the MSC behaviour at low and high oxygen tension. Figure 3 shows the results for a selected realisation.

At low oxygen the population structure is roughly regenerated by stem cells and by differentiated cells within about 1 day. At high oxygen the population is regenerated in about 2 days by stem cells but it takes about 8 days when starting with differentiated cells. This is still a surprisingly short time taking into account the large transition times for differentiated cells into the stem cell pool. This phenomenon can be understood by analysing the clone sizes of the 200 selected clones. The distributions of clone sizes after 5 days for all considered cases are shown in Figure 4. Except for regeneration from a differentiated subpopulation at high oxygen the distribution peak is located at about 50-100 cells per clone, demonstrating that most of the clones started growing. If regeneration started from differentiated cells at high oxygen, most of the cells remained quiescent throughout the observation time (137 out of 200 in Figure 4c) and only a few cells started to proliferate and formed large clones. This means the regeneration is driven by the progeny of these few cells only.

\section{Linking biomechanics and differentiation}

At the centre of expanding MSC clones proliferation becomes contact inhibited. The quiescent region grows with colony size until all cells will stop proliferation, when an expanding in vitro culture becomes confluent. Such changes in proliferation activity affect the population structure of MSC colonies. Figure 5 compares the $\alpha$-distributions of different MSC populations at high oxygen $\left(20 \% \mathrm{pO}_{2}\right)$. Shown are the $\alpha$-distributions in a low-density population without any sign of contact inhibition, in 


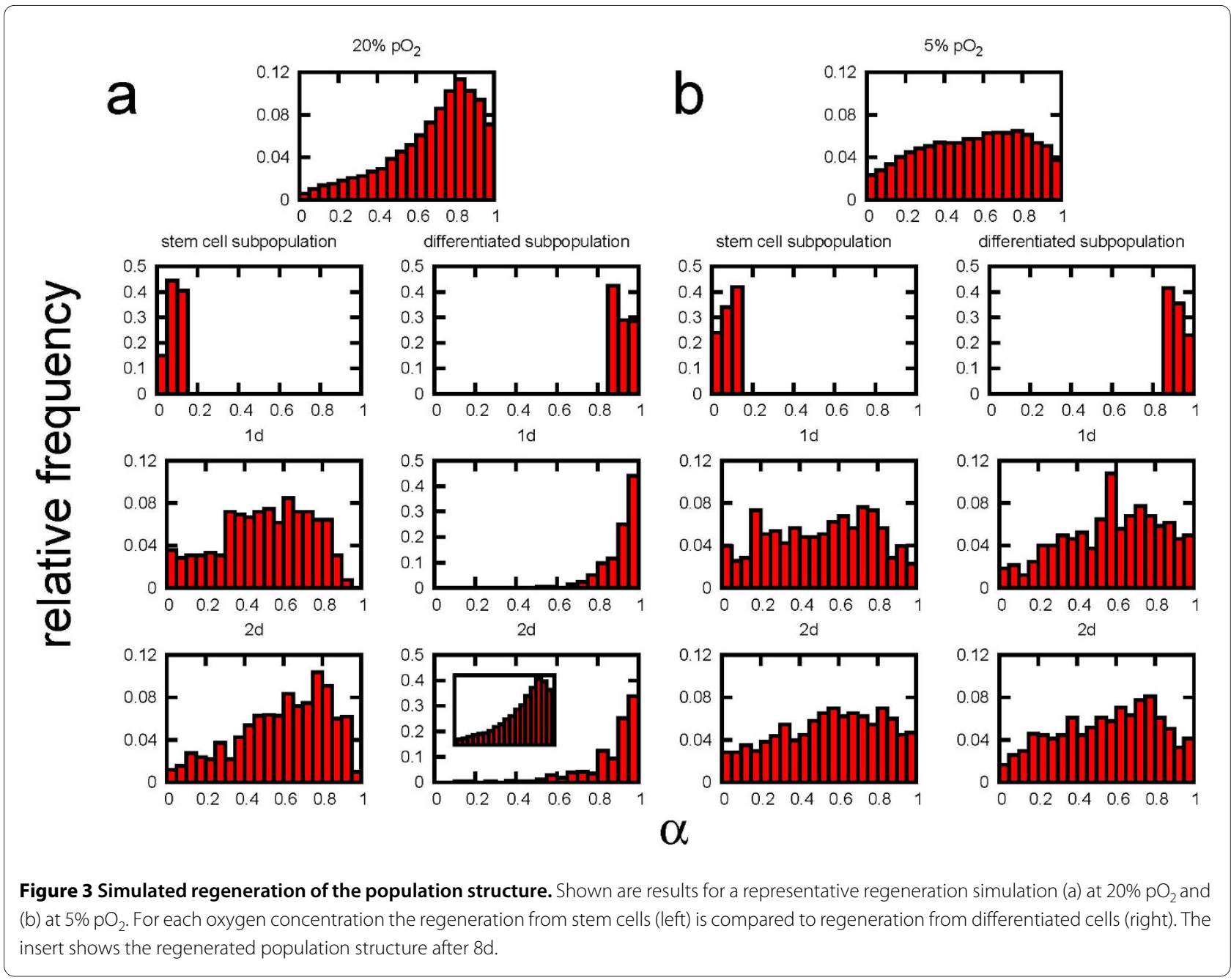

growing clones with weak and strong contact inhibition induced by variation of the cell-substrate friction con-
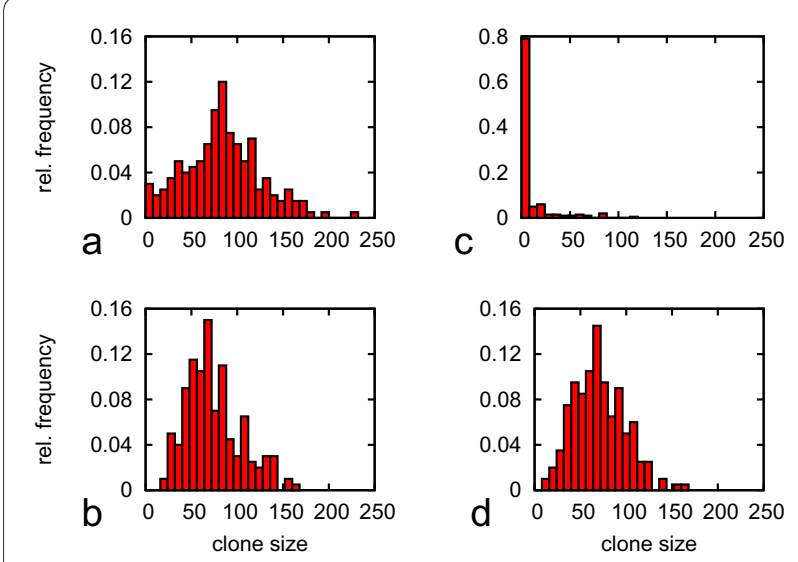

Figure 4 Simulated clonal development during the regeneration. Shown are the size distributions of 200 clones grown from stem cells $(a, b)$ and differentiated cells $(c, d)$ after 5 days of secondary culture. Upper row: $20 \% \mathrm{pO}_{2}$. Lower row: $5 \% \mathrm{pO}_{2}$. stants and in a confluent and thus quiescent population. The fraction of differentiated, non-proliferative cells $(\alpha>$ $\alpha_{d}$ ) increases from about $25 \%$ in the low density population to about $90 \%$ in the confluent population. A comparable induction of spontaneous differentiation in MSC can be observed in vitro (per. communication, A. Stolzing).

These simulation results implicate that if regeneration refers to the growth of a few large clones, as in the case of differentiated cells at high oxygen, the effect of contact inhibition becomes more relevant for population regeneration. The $\alpha$-distribution in large clones significantly differs from that of a low-density culture. Moreover, due to the increased number of differentiated cells, these populations show a lower CFU capacity (compare [14]).

\section{Modelling the organisation of the stem cell pool}

In general, 'self-renewal' of the stem cell population appears in our model as steady occupancy of stem cell states due to a particular population dynamics. Thus, additional information on MSC organisation in vitro can 


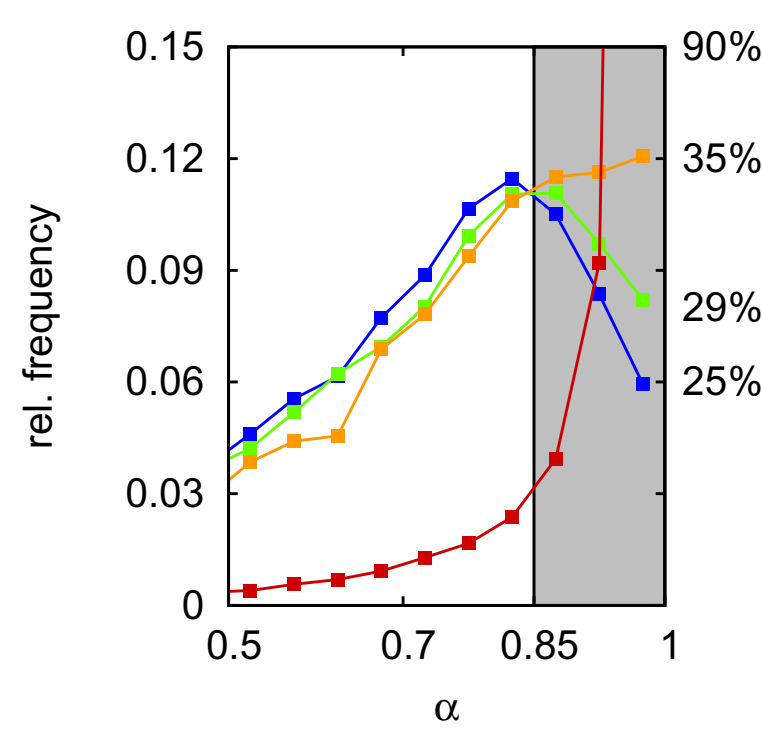

Figure $\mathbf{5} \mathrm{Impact}$ of contact inhibition of growth on regeneration. The Figure shows the equilibrium distributions of differentiation states without contact inhibition of growth (blue) and without proliferation (red) at $20 \% \mathrm{pO}_{2}$. For comparison two additional distributions obtained for populations with weak (green) and strong (yellow) contact inhibition are shown. On the right the fraction of cells in differentiated states $\left(a>a_{d}\right)$ is given. These distributions refer to regeneration experiments from differentiated cells after 7.5 days.

be obtained by performing the regeneration experiments described above in parallel for all subpopulations. Splitting the mother population into a number of subpopulations according to the expression of a differentiation marker, applying the 'regeneration protocol' suggested above to each of these subpopulations and quantifying the number of stem cells in each subpopulation after a fixed regeneration time would allow to quantify the fraction of stem cells in a MSC population descending from a particular subpopulation.

In additional simulations, we followed this concept. However, instead of splitting the mother population into subpopulations, we separated each individual cell of the mother population and followed expansion of the clones generated by the individual cells. For different time points we quantified the clonal composition of the common stem cell pool $\left(0<\alpha<\alpha_{s}=0.15\right)$ of all clones in terms of the initial $\alpha$ values of the cells that induced the clones. Figure 6 shows this clonal composition of the stem cell pool after 5 days of clonal expansion. At low oxygen (5\% $\mathrm{pO}_{2}$ ) the fraction of stem cells that originate from stem cells is about $11 \%$. At high oxygen $\left(20 \% \mathrm{pO}_{2}\right)$ this fraction decreases to only $5 \%$. In both cases, most of the cells in the stem cell pool originate from progenitor states. At low oxygen tension, all progenitor states equally contribute to this pool, while at high oxygen tension most cells origi-

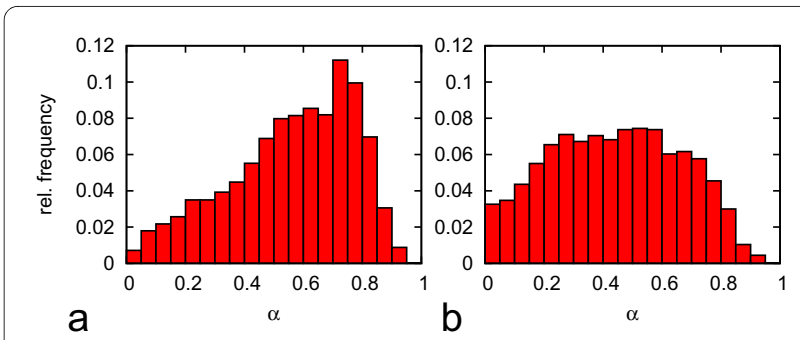

Figure 6 Simulated clonal competition in the stem cell pool. The histograms display the simulation results for the clonal composition of the stem cell pool $\left(0<a<a_{s}=0.15\right)$ for populations that were expanded at (a) $20 \%$ and (b) $5 \% \mathrm{pO}_{2}$ for 5 days.

nate from progenitor states with a high $\alpha$ value between 0.7 and 0.8 .

\section{Discussion}

Recent experimental findings indicate that cells can regain stem cell properties under defined environmental conditions. These results challenge the commonly agreed stem cell paradigm. This paradigm treats 'stemness' as a fixed property intrinsic to stem cells and assumes a deterministic and irreversible differentiation scenario for each cell [29]. As an alternative, novel concepts of functional stem cells have been developed that assign the interaction between cells and their growth environment a greater emphasis $[5,13,16]$. Treating stemness no longer as a fixed property, these concepts do not exclude certain preferred trends in the differentiation sequence, but allow reversible developments for individual cells.

We here provided the first quantitative predictions on the environmental dependent organisation of MSCs in vitro applying this novel concept. We predicted: i) the average transition times of individual cells into stem cell and differentiated states, ii) the time scales of the regeneration of the distribution of differentiation marker expression in a MSC population from subpopulations of stem and unspecific differentiated cells, and iii) the origin of the cells forming the in vitro stem cell pool of MSC. Moreover, we predicted that all these properties depend on the environment. Our results also provide estimates of the time scales of MSC adaptation to changed environmental conditions. They are in good agreement with experimental findings on MSC adaptation to low oxygen [30-32]. Particularly the work of Tang et al. [32] and Volkmer et al. [31] strengthens our modelling approach because the experimentally observed improvement of the functional competence of an entire MSC population within less than 24 hours can only hardly be explained by the expansion of residual stem cells as suggested by pedigree models.

In all our simulations, we considered an oxygen dependence of the state fluctuations. 
In contrast, biophysical features, as cell-cell and cellsubstrate interactions, were assumed to affect the regenerative potential of the MSC by interfering with their proliferation control mechanisms only. A direct feedback of these interactions on the noise amplitudes was not considered. However, recent results demonstrate that lineage specification and proliferation of MSC populations can be triggered by substrate elasticity [33] and substrate micro-structure [34]. Thus, we here suggest performing the proposed experiments on MSC plasticity on substrates that vary with respect to their elasticity and microstructure. These experiments would provide information on whether mechano-signalling can affect the kinetics of state transitions in MSCs and thus, can be used to time regeneration processes in vitro.

Our results on the composition of the stem cells pool suggest that most of the stem cells in MSC populations expanding in vitro originate from progenitor states. Thus, their mother cells underwent differentiation and de-differentiation processes and were proliferative active. Recent experimental results suggest that these cellular activities result in changes in the cellular phenotype called stem cell ageing [35]. A model that consistently describes these phenomena is currently lacking.

Most of our results could be validated by in vitro experiments on the population level. A number of suggestions were given in the text. However, more detailed studies would require tracking of individual cell fates in a single expanding MSC population. Such experiments would provide additional information on cell-cell communication in the expanding population, which was suggested to impact MSC expansion [36]. As already mentioned above, the tracking of MSC involves particular problems. Long term monitoring of MSC fates will require therefore sophisticated marker systems for both the clonal origin and the differentiation state of the cells. A number of stem cell and differentiation markers of MSC have been suggested. Good candidates are early transcription factors $[37,38]$.

Long-term fluctuations in differentiation marker expression in single cells would directly proof our concept of noise-driven stem cell organisation. For the generality of our concept, we expect such fluctuations to underlie somatic stem cell organisation independent of tissue and species.

The impact of these fluctuations may vary between different stem cell systems according to functional requirements [39]. Thus, individual stem cell systems may appear as more or less hierarchical organised. The MSC system may exhibit a pronounced flexibility, in order to be capable of instantaneous fate decisions in the course of development and in case of injury $[39,40]$.

\section{Conclusion}

Understanding single cell behaviour is prerequisite to unveil general principles of the organisation of stem cell populations. Stem cell maintenance, expansion and environmental adaptation may in particular rely on single cell plasticity. Currently only limited data on the in vitro plasticity of individual stem cells are available. We here presented for the first time quantitative simulation results on in vitro MSC plasticity applying our novel concept of noise driven stem cell differentiation. Thereby we demonstrate the suitability of the IBM approach for studying these phenomena. Challenging current views on stem cell organisation, our results predict a highly dynamic stem cell pool, whose maintenance involves permanent de-differentiation events.

\section{Authors' contributions}

AK performed all IBM simulations and following data processing. MH contributed the master equation approach, DD provided important assistance concerning IBM and ML concerning stem cells. JG did most of the intellectual contributions and major parts of draft writing. All authors have read and approved the final version of the manuscript.

\section{Acknowledgements}

The work was supported by the BMBF grant number 0313836 and $0313081 \mathrm{~F}$

\section{Author Details}

IInterdisciplinary Centre for Bioinformatics, University of Leipzig, Haertelstr. 1618, 04107 Leipzig, Germany, Institute for Medical Informatics, Statistics and Epidemiology, University of Leipzig, Haertelstr. 16-18, 04107 Leipzig, Germany and 3 French National Institute for Research in Computer Science and Control, Domaine de Voluceau-Rocquencourt, B.P. 105, 8153 Le Chesnay Cedex, France

Received: 27 October 2009 Accepted: 27 May 2010

Published: 27 May 2010

\section{References}

1. Potten CS, Loeffler M: Stem cells: attributes, cycles, spirals, pitfalls and uncertainties. Lessons for and from the crypt. Development 1990, 110:1001-1020.

2. Dingli D, Traulsen A, Michor F: (A)symmetric stem cell replication and cancer. PLoS Comput Biol 2007, 3:e53.

3. Zon LI: Intrinsic and extrinsic control of haematopoietic stem-cell selfrenewal. Nature 2008, 453:306-313.

4. Okazaki K, Maltepe E: Oxygen, epigenetics and stem cell fate. Regen Med 2006, 1:71-83

5. Loeffler M, Roeder I: Tissue stem cells: definition, plasticity, heterogeneity, self-organization and models--a conceptual approach. Cells Tissues Organs 2002, 171:8-26.

6. Zipori D: The nature of stem cells: state rather than entity. Nat Rev Genet 2004, 5:873-8.

7. Chang HH, Oh PY, Ingber DE, Huang S: Multistable and multistep dynamics in neutrophil differentiation. BMC Cell Biol 2006, 7:1 1.

8. Chang $\mathrm{HH}$, Hemberg M, Barahona M, Ingber DE, Huang S: Transcriptomewide noise controls lineage choice in mammalian progenitor cells. Nature 2008, 453:544-547.

9. Hunziker EB: Articular cartilage repair: basic science and clinical progress. A review of the current status and prospects. Osteoarthritis Cartilage 2002, 10:432-463.

10. Pontikoglou C, Delorme B, Charbord P: Human bone marrow native mesenchymal stem cells. Regen Med 2008, 3:731-41.

11. Sparks RL, Seibel-Ross El, Wier ML, Scott RE: Differentiation, dedifferentiation, and transdifferentiation of BALB/C $3 T 3 \mathrm{~T}$ mesenchymal stem cells: potential significance in metaplasia and neoplasia. Cancer Res 1986, 46:5312-9. 
12. Song $L$, Webb NE, Song $Y$, Tuan RS: Identification and functional analysis of candidate genes regulating mesenchymal stem cell self-renewal and multipotency. Stem Cells 2006, 24:1707-1718.

13. Zipori D: The stem state: mesenchymal plasticity as a paradigm. Curr Stem Cell Res Ther 2006, 1:95-102.

14. Krinner A, Zscharnack M, Bader A, Drasdo D, Galle J: The impact of the oxygen environment on mesenchymal stem cell expansion and chondrogenic differentiation. Cell Proliferation 2009, 42:471-484.

15. Hoffmann M, Chang HH, Huang S, Ingber DE, Loeffler M, Galle J: Noisedriven stem cell and progenitor population dynamics. PLOS ONE 2008 3:e2922.

16. Loeffler M, Roeder I: Conceptual models to understand tissue stem cell organization. Curr Opin Hematol 2004, 11:81-87.

17. Lécuyer E, Yoshida H, Parthasarathy N, Alm C, Babak T, Cerovina T, Hughes TR, Tomancak P, Krause HM: Global analysis of mRNA localization reveals a prominent role in organizing cellular architecture and function. Cell 2007, 131:174-187.

18. Brown G, Drayson MT, Durham J, Toellner KM, Hughes PJ, Choudhry MA, Taylor DR, Bird R, Michell RH: HL60 cells halted in G1 or S phase differentiate normally. Exp Cell Res 2002, 281:28-38.

19. Delorme B, Ringe J, Pontikoglou C, Gaillard J, Langonne A, Sensebe L, Noel D, Jorgensen C, Haupl T, Charbord P: Specific lineage-priming of bone marrow mesenchymal stem cells provides the molecular framework for their plasticity. Stem Cells 2009, 27:1142-51.

20. Zscharnack M, Poesel C, Galle J, Bader A: Low Oxygen Expansion Improves Subsequent Chondrogenesis of Ovine Bone-MarrowDerived Mesenchymal Stem Cells in Collagen Type I Hydrogel. Cells Tissues Organs 2009, 190:81-93.

21. Galle J, Loeffler M, Drasdo D: Modeling the effect of deregulated proliferation and apoptosis on the growth dynamics of epithelial cell populations in vitro. Biophys $J$ 2005, 88:62-75.

22. Landau L, Lifschitz E: Theory of Elasticity, 3.Rev. Pergamon Press, Oxford; 1986

23. Drasdo $D$, Hoehme $S$ : A single-cell-based model of tumor growth in vitro: monolayers and spheroids. Phys Biol 2005, 2:133-147.

24. Van Kampen NG: Stochastic Processes in Physics and Chemistry. 2nd edition. Elsevier; 2007

25. Eilken HM, Nishikawa SI, Schroeder T: Continuous single-cell imaging of blood generation from haemogenic endothelium. Nature 2009, 457:896-900.

26. Lo Celso C, Fleming HE, Wu JW, Zhao CX, Miake-Lye S, Fujisaki J, Côté D, Rowe DW, Lin CP, Scadden DT: Live-animal tracking of individual haematopoietic stem/progenitor cells in their niche. Nature 2009 457:92-96.

27. Christley S, Alber MS, Newman SA: Patterns of mesenchymal condensation in a multiscale, discrete stochastic model. PLoS Comput Biol 2007, 3:e76.

28. DeLise AM, Fischer L, Tuan RS: Cellular interactions and signaling in cartilage development. Osteoarthritis Cartilage 2000, 8:309-334

29. Reya T, Morrison SJ, Clarke MF, Weissman IL: Stem cells, cancer, and cancer stem cells. Nature 2001, 414:105-111.

30. Martin-Rendon E, Hale SJ, Ryan D, Baban D, Forde SP, Roubelakis M, Sweeney D, Moukayed M, Harris AL, Davies K, et al:: Transcriptional profiling of human cord blood CD133+ and cultured bone marrow mesenchymal stem cells in response to hypoxia. Stem Cells 2007, 25:1003-12.

31. Volkmer E, Kallukalam BC, Maertz J, Otto S, Drosse I, Polzer H, Bocker W, Stengele M, Docheva D, Mutschler W, et al.: Hypoxic preconditioning of human mesenchymal stem cells overcomes hypoxia-induced inhibition of osteogenic differentiation. Tissue Eng Part A 2010 16:153-64.

32. Tang YL, Zhu W, Cheng M, Chen L, Zhang J, Sun T, Kishore R, Phillips MI, Losordo DW, Qin G: Hypoxic preconditioning enhances the benefit of cardiac progenitor cell therapy for treatment of myocardial infarction by inducing CXCR4 expression. Circ Res 2009, 104:1209-16

33. Engler AJ, Sen S, Sweeney HL, Discher DE: Matrix elasticity directs stem cell lineage specification. Cell 2006, 126:677-689.

34. Dalby MJ, Gadegaard N, Tare R, Andar A, Riehle MO, Herzyk P, Wilkinson CDW, Oreffo ROC: The control of human mesenchymal cell differentiation using nanoscale symmetry and disorder. Nat Mater 2007, 6:997-1003.
35. Stolzing A, Scutt A: Age-related impairment of mesenchymal progenitor cell function. Aging Cell 2006, 5:213-224.

36. Gregory CA, Ylostalo J, Prockop DJ: Adult bone marrow stem/progenitor cells (MSCs) are preconditioned by microenvironmental "niches" in culture: a two-stage hypothesis for regulation of MSC fate. SCi STKE 2005, 2005:pe37.

37. Lefebvre V, Smits P: Transcriptional control of chondrocyte fate and differentiation. Birth Defects Res. CEmbryo Today 2005, 75:200-212.

38. Komori T: Regulation of osteoblast differentiation by transcription factors. J Cell Biochem 2006, 99:1233-1239.

39. Zipori D: The stem state: plasticity is essential, whereas self-renewal and hierarchy are optional. Stem Cells 2005, 23:719-26.

40. Kuhn NZ, Tuan RS: Regulation of stemness and stem cell niche of mesenchymal stem cells: implications in tumorigenesis and metastasis. J Cell Physiol 2010, 222:268-77.

doi: 10.1186/1752-0509-4-73

Cite this article as: Krinner et al., Individual fates of mesenchymal stem cells in vitro BMC Systems Biology 2010, 4:73

\section{Submit your next manuscript to BioMed Central and take full advantage of:}

- Convenient online submission

- Thorough peer review

- No space constraints or color figure charges

- Immediate publication on acceptance

- Inclusion in PubMed, CAS, Scopus and Google Scholar

- Research which is freely available for redistribution
C Biomed Central 